\title{
Morphological Characterization and Identification of Morphological Markers for Selected Sugarcane (Saccharum spp.) Cultivars
}

\author{
K. Chidambaram ${ }^{1 *}$ and K. Sivasubramaniam ${ }^{2}$ \\ ${ }^{1}$ Department of Seed Science and Technology, TNAU, Coimbatore, Tamil Nadu, India \\ ${ }^{2}$ Agricultural College and Research Institute, Pudukkottai, Tamil Nadu, India \\ *Corresponding author
}

\section{A B S T R A C T}

\section{Keywords \\ Sugarcane, Sugarcane cultivars, Varietal identification, Morphological characters, Morphological markers. \\ Article Info \\ Accepted: \\ 07 October 2017 Available Online: 10 December 2017}

The variety identification serves the important goals such as mitigating legal claims and confirming intellectual property rights and maintenance of genetic purity. Plant morphological characters are universally adopted descriptors for DUS testing and varietal characterization of crop genotypes. In this study 10 sugarcane cultivars (TNAU Sugarcane Si. 6, TNAU Sugarcane Si. 7, TNAU Sugarcane Si. 8, Co 86032, Co 99004, Co 99006, Co 94008, Co 94012, Co 2001-13 and Co 2001-15) were characterized using 27 morphological descriptors. Among the 27 descriptors, width of root band opposite to bud alone was monomorphic, 14 were dimorphic and 12 were found to be polymorphic. Distinct morphological profiles were obtained for four out of ten cultivars. The dendrogram based on UPGMA analysis using DUS characters, grouped ten sugarcane cultivars into two major groups at 43 per cent similarity level. The similarity matrix coefficient ranged from 43 per cent to 63 per cent with an average of 53 per cent.

\section{Introduction}

Sugarcane (Saccharum spp.) is one of the most important industrial crops in the world. The leading sugarcane producing countries of the world are Brazil, India, China, and Thailand. Cane sugar constitutes around 80 per cent of the total sugar produced in the world and the rest being contributed by beet sugar and other sources such as hydrolyzed starch product. Sugarcane cultivars differ in their agronomic traits like; stalk height, girth and sugar contents due to their genetic makeup and management practices. Different sugarcane cultivars generally resemble each other in their appearance, but each has different morphological characters. Among the characters that are generally influenced by environmental factors are usually quantitative character like size, number and colour, etc. These characters are not as valuable in identifying a variety as are stable characters, like the shape of vegetative organs and arrangement of various floret parts (Grassl, 1956). Many attempts have been made to define the morphological characteristics for identification of different sugarcane cultivars (Barber, 1915; Cowgill, 1917). These characteristics are the size, number and colour of stalks, bud, node and nodal characteristics, ivory markings, splits, bud groove, leaf characteristics, adult root system and 
underground branching. A botanical description is a necessity for sugarcane workers to enable them to identify the cultivars in the field. The knowledge of morphology will help in identification and characterization of clones, cultivars, cultivars and related species. The main objective of the present study was to characterize the selected sugarcane cultivars to provide the information about morphological markers that help in the identification of different sugarcane cultivars in the field.

\section{Materials and Methods}

\section{Material for varietal identification}

In the present study, setts belonging to three sugarcane cultivars from Sugarcane Research Station, Sirugamani (TNAU Sugarcane Si. 6, TNAU Sugarcane Si. 7, TNAU Sugarcane Si. 8 ) and seven cultivars from Sugarcane Breeding Institute, Coimbatore (Co 86032, Co 99004, Co 99006, Co 94008, Co 94012, Co 2001-13 and Co 2001-15) were used for conducting research work.

\section{Study of plant morphological traits and} observations recorded

The sets of ten sugarcane cultivars (Table 1) were sown in the field of Agricultural College and Research Institute, Madurai during July 2012. All the recommended agronomic and plant protection measures were adopted for raising a healthy crop. The DUS guidelines of the Protection of Plant Varieties and Farmers' Rights Authority (PPV and FRA), India was used to characterize sugarcane cultivars.

\section{Results and Discussion}

In the present study, ten cultivars of sugarcane viz., TNAU Sugarcane Si. 6, TNAU Sugarcane Si. 7, TNAU Sugarcane Si. 8, Co 86032, Co 99004, Co 99006, Co 94008,
Co 94012, Co 2001-13 and Co 2001-15, where characterized using 27 morphological DUS descriptors as prescribed by DUS guidelines of PPV and FR Authority, New Delhi, India. The morphological trait observations were taken at three different stages of crop growth, which were 240 (End of grand growth stage), 300 (Maturity stage) and 360 (Harvest stage) days after planting. Among the 27 descriptors, width of roots band opposite to bud alone was monomorphic, 14 were dimorphic and 12 were found to be polymorphic (Table 2). The dendrogram based on UPGMA cluster analysis using DUS characters, grouped ten sugarcane cultivars into two major Groups at 43 per cent similarity level (Fig. 1). The similarity matrix coefficient ranged from 43 per cent to 63 per cent with an average of 53 per cent. In Group 1, genotype Co 86032 alone formed a separate cluster in the phonogram. In Group 2, there were 2 major subgroups. Sub group 1 contained cultivars TNAU Sugarcane Si. 7, TNAU Sugarcane Si. 8 and Co 94012 were genetically close to each other. Sub group 2 included 6 cultivars i.e., TNAU Sugarcane Si. 6, Co 94008, Co 99006, Co 2001-15, Co 99004 and Co 200113 showing more genetic similarities.

A botanical description is a necessity for sugarcane workers to enable them to identify the cultivars in the field. The knowledge of morphology will help in identification and characterization of clones, cultivars, cultivars and related species. In the present study, distinct morphological profiles were obtained for four out of ten cultivars that help in the identification of different sugarcane cultivars in the field, i.e., erect plant growth habit in Co 99004 (Fig. 2), Smooth Internode rind surface and long lanceolate inner auricle in TNAU Sugarcane Si. 8 (Fig. 3), Tight clasping of leaf sheaths on internodes in Co 2001-13 (Fig. 4) and Light internode waxiness and weak (not swollen) growth ring in Co 86032 (Fig. 5). 
Fig.1 Dendrogram depicting the classification of ten sugarcane cultivars constructed through UPGMA method and based on morphological markers

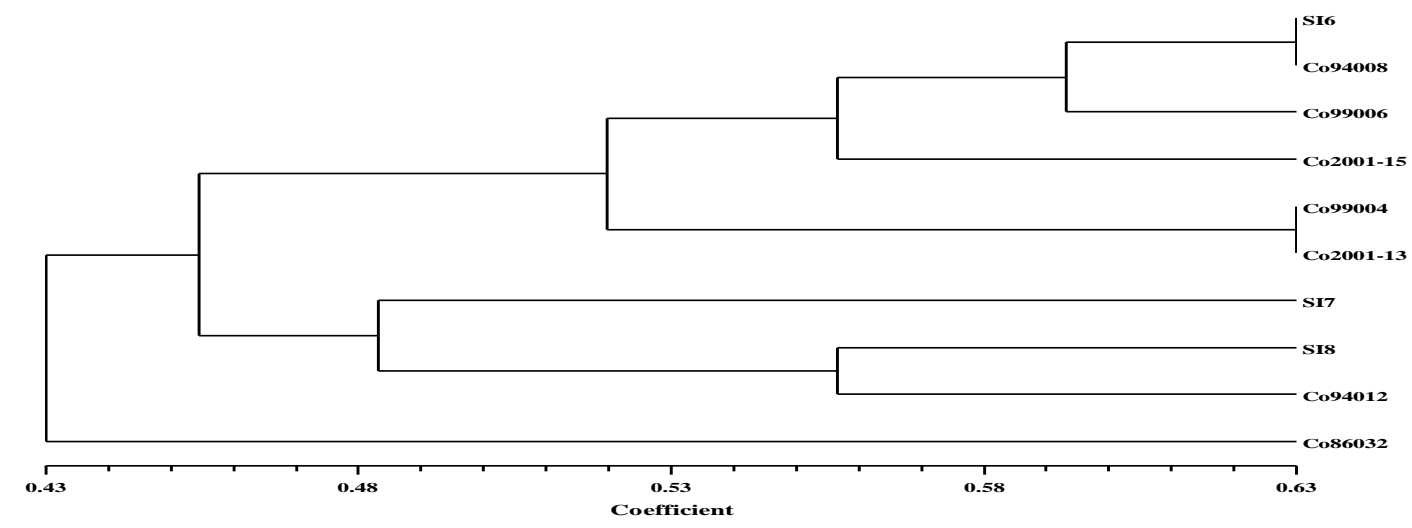

Fig.2 Distinct morphological profile of Co 99004 (erect plant growth habit)

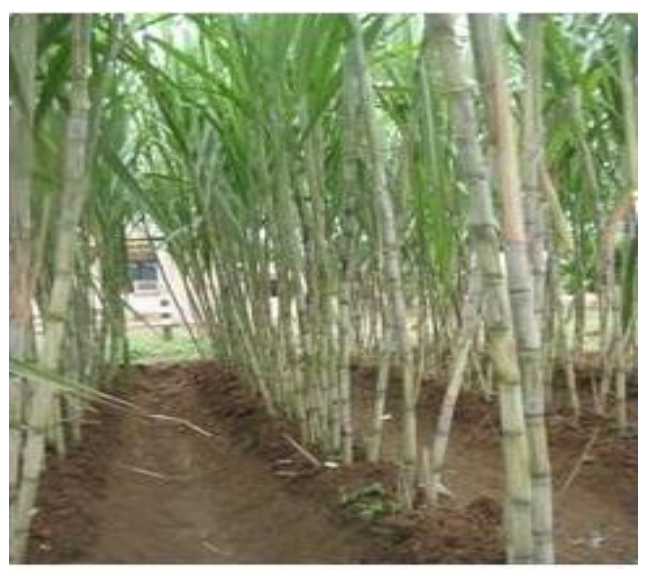

Co 99004

Fig.3 Distinct morphological profile of TNAU Sugarcane Si. 8 (long lanceolate inner auricle and smooth Internode rind surface)

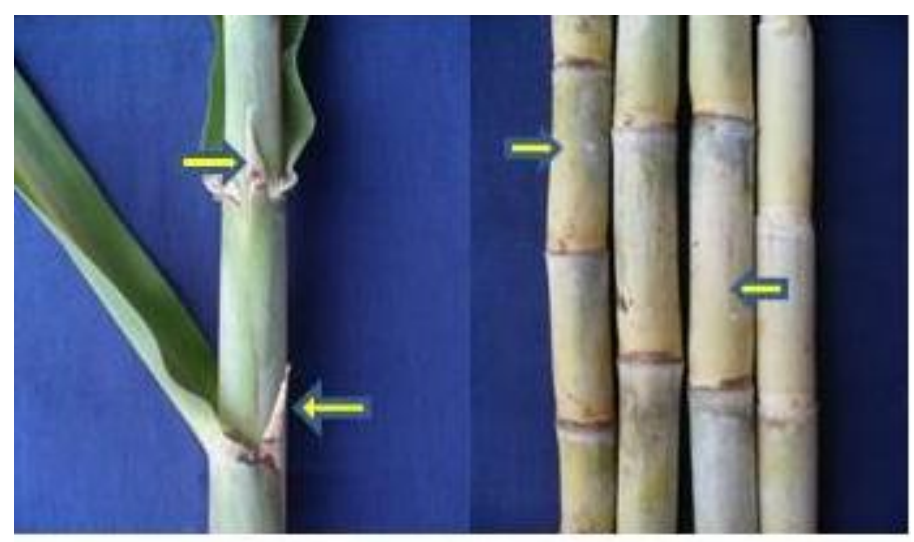

TNAU Sugarcane Si. 8 
Fig.4 Distinct morphological profile of Co 2001-13 (tight clasping of leaf sheaths on internodes)

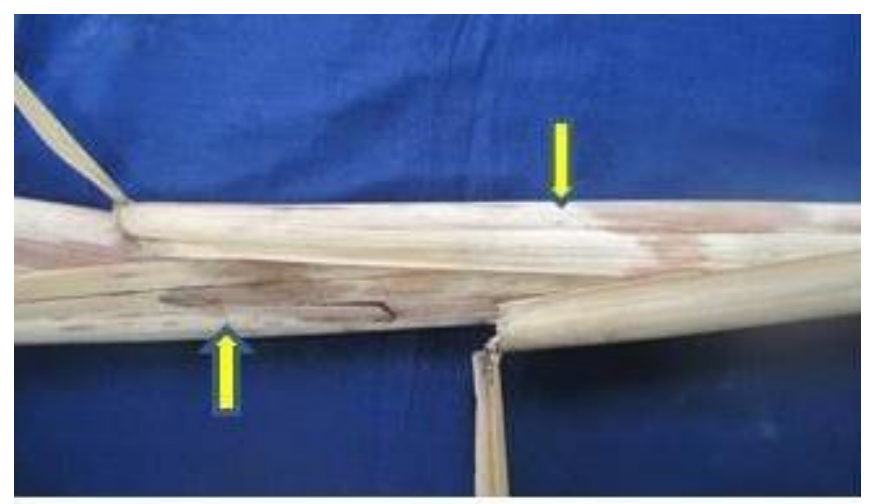

Co 2001-13

Fig.5 Distinct morphological profile of Co 86032 (light internode waxiness and weak (not swollen) growth ring)

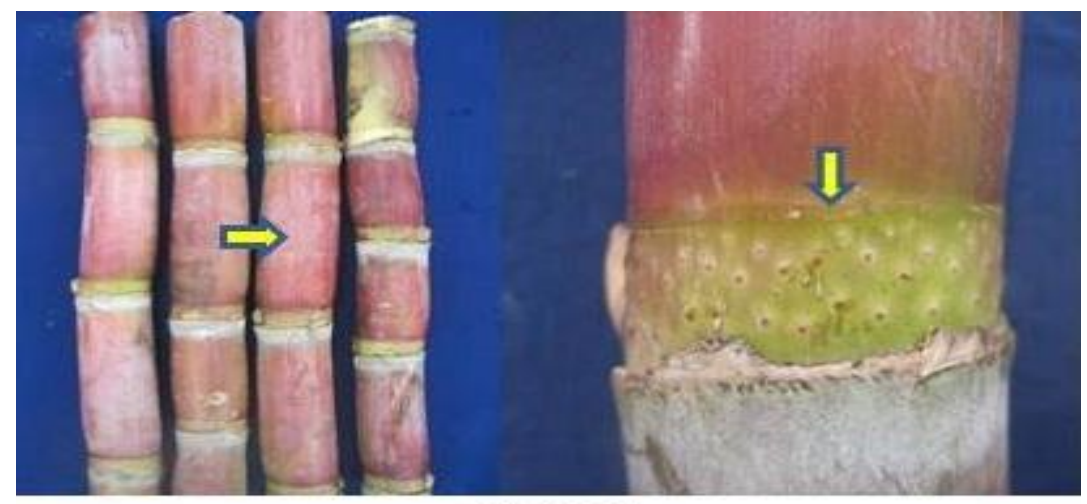

Co 86032

Table.1 Sugarcane cultivars subjected to varietal characterization

\begin{tabular}{|c|c|c|c|c|}
\hline $\begin{array}{c}\text { S. } \\
\text { No. }\end{array}$ & Variety & Parentage & $\begin{array}{c}\text { Origin/ } \\
\text { Release Center }\end{array}$ & $\begin{array}{c}\text { Duration } \\
\text { (Month) }\end{array}$ \\
\hline 1 & TNAU Sugarcane Si. 6 & Co $8213 \times$ CoA 7602 & Sirugamani & 12 \\
\hline 2 & TNAU Sugarcane Si. 7 & Co 99034 x Co.G. 93076 & Sirugamani & 12 \\
\hline 3 & TNAU Sugarcane Si. 8 & CoC $90063 \times$ Co 8213 & Sirugamani & 12 \\
\hline 4 & Co 86032 & Co $62198 \times$ CoC 671 & SBI & 12 \\
\hline 5 & Co 99004 & Co $62175 \times$ Co 86250 & SBI & 12 \\
\hline 6 & Co 99006 & 84 WL $22 \times$ Co 775 & SBI & 12 \\
\hline 7 & Co 94008 & Co $7201 \times$ Co 775 & SBI & 12 \\
\hline 8 & Co 94012 & Somaclone of CoC 671 & SBI & 12 \\
\hline 9 & Co $2001-13$ & Co 7806 GC & SBI & 12 \\
\hline 10 & Co $2001-15$ & C0 $85002 \times$ Co 775 & SBI & 12 \\
\hline
\end{tabular}


Table.2 Morphological characterization of sugarcane cultivars based on DUS guidelines

\begin{tabular}{|c|c|c|c|c|}
\hline $\begin{array}{l}\text { S. } \\
\text { No }\end{array}$ & Characteristics & States & Cultivars & Score \\
\hline \multirow[t]{2}{*}{1} & \multirow{2}{*}{$\begin{array}{l}* * \text { Plant: } \\
\text { Growth habit }\end{array}$} & Erect & Co 99004 & 1 \\
\hline & & Semi-erect & $\begin{array}{c}\text { TNAU Sugarcane Si. 6, TNAU Sugarcane Si. 7, } \\
\text { TNAU Sugarcane Si 8, Co 86032, Co 99006, } \\
\text { Co 94008, Co 94012, Co 2001-13 } \\
\text { and Co 2001-15. }\end{array}$ & 2 \\
\hline \multirow[t]{3}{*}{2} & \multirow{3}{*}{$\begin{array}{c}\text { ****Leaf } \\
\text { sheath:Hairiness }\end{array}$} & Absent & TNAU Sugarcane Si. 7, Co 99004 and Co 99006. & 1 \\
\hline & & Sparse & $\begin{array}{l}\text { TNAU Sugarcane Si. 6, TNAU Sugarcane Si. 8, } \\
\text { Co 86032, Co 94008 and Co 2001-13. }\end{array}$ & 3 \\
\hline & & Dense & Co 94012 and Co 2001-15 & 5 \\
\hline \multirow[t]{4}{*}{3} & \multirow[t]{4}{*}{$\begin{array}{l}* * * \text { Leaf sheath: } \\
\text { Shape of ligule }\end{array}$} & $\begin{array}{l}\text { Strap- } \\
\text { shaped }\end{array}$ & Co 99004 & 1 \\
\hline & & Deltoid & $\begin{array}{c}\text { TNAU Sugarcane Si. 6, TNAU Sugarcane Si. } 7 \\
\text { and Co 94012. }\end{array}$ & 2 \\
\hline & & $\begin{array}{l}\text { Crescent- } \\
\text { shaped }\end{array}$ & $\begin{array}{l}\text { TNAU Sugarcane Si. 8, Co 86032, Co 99006, } \\
\text { Co 94008, Co 2001-13 and Co 2001-15. }\end{array}$ & 3 \\
\hline & & $\begin{array}{l}\text { Arch (bow) } \\
\text { shaped }\end{array}$ & $-1+--$ & 4 \\
\hline \multirow[t]{7}{*}{4} & \multirow{7}{*}{$\begin{array}{l}* * * \text { Leaf sheath: } \\
\text { Shape of inner } \\
\text { Auricle }\end{array}$} & Incipient & TNAU Sugarcane Si. 7 and Co 94008 & 1 \\
\hline & & Deltoid & Co 86032, Co 99004, Co 99006 and Co 2001-13. & 2 \\
\hline & & Dentoid & TNAU Sugarcane Si. 6 & 3 \\
\hline & & Unciform & ---- & 4 \\
\hline & & Calcariform & Co 2001-15 & 5 \\
\hline & & Lanceolate & TNAU Sugarcane Si. 8 and Co 94012. & 6 \\
\hline & & Falcate & ---- & 7 \\
\hline \multirow[t]{6}{*}{5} & \multirow[t]{6}{*}{$\begin{array}{l}* * * \text { Leaf sheath: } \\
\text { Colour of } \\
\text { dewlap }\end{array}$} & $\begin{array}{c}\text { Green } \\
(\text { PMS 367, } \\
373,374)\end{array}$ & Co 2001-13, Co 99004 and Co 86032. & 1 \\
\hline & & $\begin{array}{c}\text { Greenish- } \\
\text { Yellow } \\
\text { (PMS 381, } \\
\text { 387) } \\
\end{array}$ & Co 2001-15 and TNAU Sugarcane Si. 8 & 2 \\
\hline & & Yellow & ---- & 3 \\
\hline & & $\begin{array}{l}\text { Yellowish } \\
\text { green } \\
\text { (PMS 100, } \\
\text { 393) }\end{array}$ & TNAU Sugarcane Si. 6 and TNAU Sugarcane Si. 7 & 4 \\
\hline & & Brown & $-1+--$ & 5 \\
\hline & & $\begin{array}{c}\text { Purple } \\
\text { (PMS } \\
224,225\end{array}$ & Co 94008, Co 94012 and Co 99006. & 6 \\
\hline
\end{tabular}




\begin{tabular}{|c|c|c|c|c|}
\hline & & 231) & & \\
\hline \multirow[t]{3}{*}{6} & \multirow{3}{*}{$\begin{array}{l}* * * \text { Leaf blade: } \\
\text { Curvature }\end{array}$} & Erect & TNAU Sugarcane Si. 7 and Co 86032. & 1 \\
\hline & & Curved tip & Co 99004, Co 2001-13 and Co 2001-15. & 2 \\
\hline & & Arched & $\begin{array}{l}\text { TNAU Sugarcane Si. 6, TNAU Sugarcane Si. 8, } \\
\text { Co } 99006, \text { Co } 94008 \text { and Co } 94012 .\end{array}$ & 3 \\
\hline \multirow[t]{3}{*}{7} & \multirow[t]{3}{*}{$\begin{array}{l}\text { ** Leaf blade: } \\
\text { Width }\end{array}$} & $\begin{array}{c}\text { Narrow } \\
(<3.0 \mathrm{~cm})\end{array}$ & ---- & 3 \\
\hline & & $\begin{array}{l}\text { Medium } \\
(3.0-5.0 \\
\mathrm{cm})\end{array}$ & $\begin{array}{l}\text { TNAU Sugarcane Si. 7, TNAU Sugarcane Si. } 8 \text { and } \\
\text { Co 2001-15. }\end{array}$ & 5 \\
\hline & & $\begin{array}{c}\text { Broad } \\
(>5.0 \mathrm{~cm})\end{array}$ & $\begin{array}{l}\text { TNAU Sugarcane Si. 6, Co } 86032 \text {, Co } 99004 \text {, } \\
\text { Co } 99006 \text {, Co } 94008 \text {, Co } 94012 \text { and Co 2001-13. }\end{array}$ & 7 \\
\hline \multirow[t]{3}{*}{8} & \multirow[t]{3}{*}{$\begin{array}{l}\text { ***Plant: } \\
\text { Adherence of } \\
\text { leaf sheath }\end{array}$} & $\begin{array}{l}\text { Weak } \\
\text { (self de- } \\
\text { trashing) }\end{array}$ & $\begin{array}{l}\text { TNAU Sugarcane Si. 6, TNAU Sugarcane Si. 8, } \\
\text { Co 86032, Co } 99004 \text {, Co 99006, Co 94008, Co } \\
94012 \text { and Co 2001-15. }\end{array}$ & 3 \\
\hline & & $\begin{array}{l}\text { Medium } \\
\text { (semi } \\
\text { clasping) }\end{array}$ & $\begin{array}{l}\text { TNAU Sugarcane Si. } 7 \\
\end{array}$ & 5 \\
\hline & & $\begin{array}{l}\text { Strong } \\
\text { (tight } \\
\text { clasping) }\end{array}$ & Co 2001-13 & 7 \\
\hline \multirow[t]{9}{*}{9} & \multirow{9}{*}{$\begin{array}{l}* * * \text { Internode: } \\
\text { Colour (Not } \\
\text { exposed to sun) }\end{array}$} & Green & ---- & 1 \\
\hline & & $\begin{array}{c}\text { Green } \\
\text { yellow } \\
\text { (PMS 380, } \\
381,382 \text { ) }\end{array}$ & Co 2001-15, TNAU Sugarcane Si. 8 and Co 99006. & 2 \\
\hline & & $\begin{array}{l}\text { Green } \\
\text { white }\end{array}$ & --- & 3 \\
\hline & & $\begin{array}{c}\text { Yellow } \\
(\text { PMS } \\
1205,382)\end{array}$ & TNAU Sugarcane Si. 7 and Co 99004 & 4 \\
\hline & & $\begin{array}{c}\text { Yellow } \\
\text { green } \\
\text { (PMS 122, } \\
379,394 \text {, } \\
395 \text { ) }\end{array}$ & $\begin{array}{l}\text { Co 94012, TNAU Sugarcane Si. 6, Co 2001-13, } \\
\text { Co } 86032 \text { and Co } 94008 .\end{array}$ & 5 \\
\hline & & $\begin{array}{l}\text { Yellow } \\
\text { white }\end{array}$ & --- & 6 \\
\hline & & $\begin{array}{l}\text { Orange } \\
\text { white }\end{array}$ & ---- & 7 \\
\hline & & $\begin{array}{c}\text { Greyed } \\
\text { green }\end{array}$ & ---- & 8 \\
\hline & & $\begin{array}{l}\text { Greyed } \\
\text { yellow }\end{array}$ & --- & 9 \\
\hline 10 & $\begin{array}{l}* * * \text { Internode: } \\
\text { Colour }\end{array}$ & $\begin{array}{l}\text { Green } \\
\text { yellow }\end{array}$ & TNAU Sugarcane Si. 8 & 1 \\
\hline
\end{tabular}




\begin{tabular}{|c|c|c|c|c|}
\hline & $\begin{array}{l}\text { (Exposed to } \\
\text { sun) }\end{array}$ & $\begin{array}{l}\text { group } \\
(\mathrm{PMS} \\
3822 \mathrm{x})\end{array}$ & & \\
\hline & & $\begin{array}{c}\begin{array}{c}\text { Yellow } \\
\text { green } \\
\text { group }\end{array} \\
\text { (PMS 388, } \\
\text { 3965) }\end{array}$ & TNAU Sugarcane Si. 6 and Co 99004 & 2 \\
\hline & & $\begin{array}{l}\text { Yellow } \\
\text { group } \\
\text { (PMS 396) }\end{array}$ & Co 99006 & 3 \\
\hline & & $\begin{array}{l}\text { Greyed } \\
\text { group }\end{array}$ & ---- & 4 \\
\hline & & $\begin{array}{l}\text { Brown } \\
\text { group } \\
\text { (PMS 702, } \\
703)\end{array}$ & Co 86032 and Co 2001-15 & 5 \\
\hline & & $\begin{array}{c}\text { Purple } \\
\text { group } \\
\text { (PMS 205, } \\
210,212, \\
215)\end{array}$ & $\begin{array}{l}\text { Co 94012, TNAU Sugarcane Si. 7, Co 2001-13 and } \\
\text { Co } 94008 .\end{array}$ & 6 \\
\hline 11 & $\begin{array}{l}* * \text { Internode: } \\
\text { Diameter }\end{array}$ & $\begin{array}{l}\text { Thin } \\
(<2.2 \mathrm{~cm})\end{array}$ & ---- & 3 \\
\hline & & $\begin{array}{l}\text { Medium } \\
(2.2-3.0 \\
\mathrm{cm})\end{array}$ & $\begin{array}{l}\text { TNAU Sugarcane Si. 6, Co 99004, Co 2001-13 and } \\
\text { Co 2001-15. }\end{array}$ & 5 \\
\hline & & $\begin{array}{l}\text { Thick } \\
(>3.0 \mathrm{~cm})\end{array}$ & $\begin{array}{l}\text { TNAU Sugarcane Si. 7, TNAU Sugarcane Si. 8, Co } \\
\text { 86032, Co 99006, Co } 94008 \text { and Co } 94012 \text {. }\end{array}$ & 7 \\
\hline 12 & $\begin{array}{l}\text { **Internode: } \\
\text { Shape }\end{array}$ & Cylindrical & $\begin{array}{l}\text { TNAU Sugarcane Si. 6, TNAU Sugarcane Si. 7, } \\
\text { TNAU Sugarcane Si. 8, Co 99004, Co 99006, Co } \\
\text { 2001-13 and Co 2001-15. }\end{array}$ & 1 \\
\hline & & Tumescent & --- & 2 \\
\hline & & $\begin{array}{l}\text { Bobbin } \\
\text { shaped }\end{array}$ & ---- & 3 \\
\hline & & Conoidal & Co 86032 , Co 94008 and Co 94012. & 4 \\
\hline & & Obconoidal & $\begin{array}{c}---- \\
\end{array}$ & 5 \\
\hline & & Curved & ---- & 6 \\
\hline 13 & $\begin{array}{l}* * \text { Internode: } \\
\text { Zig zag }\end{array}$ & Absent & $\begin{array}{l}\text { TNAU Sugarcane Si. 7, TNAU Sugarcane Si. 8, Co } \\
\qquad 86032 \text { and Co } 94012 .\end{array}$ & 1 \\
\hline & alignment & Present & $\begin{array}{l}\text { TNAU Sugarcane Si. 6, Co 99004, Co 99006, Co } \\
\text { 94008, } \\
\text { Co 2001-13 and Co 2001-15. }\end{array}$ & 9 \\
\hline 14 & $\begin{array}{l}\text { **Internode: } \\
\text { Growth crack } \\
\quad \text { (Split) }\end{array}$ & Absent & $\begin{array}{l}\text { TNAU Sugarcane Si. 7, TNAU Sugarcane Si. 8, Co } \\
\text { 99004, Co 99006, Co 94008, Co } 94012 \text { and Co } \\
\text { 2001-13. }\end{array}$ & 1 \\
\hline
\end{tabular}




\begin{tabular}{|c|c|c|c|c|}
\hline & & Present & TNAU Sugarcane Si. 6, Co 86032 and Co 2001-15. & 9 \\
\hline \multirow[t]{4}{*}{15} & \multirow{4}{*}{$\begin{array}{l}* * * \text { Internode: } \\
\text { Rind surface } \\
\text { appearance }\end{array}$} & Smooth & TNAU Sugarcane Si. 8 & 1 \\
\hline & & $\begin{array}{l}\text { Corky } \\
\text { patches } \\
\text { only }\end{array}$ & $\begin{array}{l}\text { TNAU Sugarcane Si. 6, Co 99004, Co 99006, Co } \\
\text { 94012, } \\
\text { Co 2001-13 and Co 2001-15. }\end{array}$ & 2 \\
\hline & & $\begin{array}{c}\text { Ivory } \\
\text { marks only }\end{array}$ & TNAU Sugarcane Si. 7 & 3 \\
\hline & & $\begin{array}{l}\text { Corky } \\
\text { patches and } \\
\text { ivory marks } \\
\text { present }\end{array}$ & Co 86032 and Co 94008 & 4 \\
\hline \multirow[t]{3}{*}{16} & \multirow{3}{*}{$\begin{array}{l}* * * \text { Internode: } \\
\text { waxiness }\end{array}$} & Light & Co 86032 & 3 \\
\hline & & Medium & $\begin{array}{l}\text { TNAU Sugarcane Si. 6, TNAU Sugarcane Si. 7, } \\
\text { TNAU Sugarcane Si. 8, Co 99006, Co } 94012 \text { and } \\
\text { Co 2001-15. }\end{array}$ & 5 \\
\hline & & Heavy & Co 99004, Co 94008 and Co 2001-13. & 7 \\
\hline \multirow[t]{9}{*}{17} & \multirow[t]{9}{*}{$\begin{array}{l}* * * \text { Node: shape } \\
\text { of bud }\end{array}$} & Ovate & $\begin{array}{l}\text { TNAU Sugarcane Si. 6, Co 86032, Co 99004, Co } \\
\text { 99006, } \\
\text { Co } 94008 \text { and Co 2001-15. }\end{array}$ & 1 \\
\hline & & Obovate & 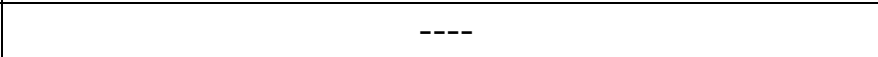 & 2 \\
\hline & & Oval & Co 94012 & 3 \\
\hline & & Round & $\begin{array}{l}\text { TNAU Sugarcane Si. 7, TNAU Sugarcane Si. } 8 \\
\text { and } \\
\text { Co 2001-13. }\end{array}$ & 4 \\
\hline & & Pentagonal & ---- & 5 \\
\hline & & Rhomboid & ---- & 6 \\
\hline & & Rectangular & $\begin{array}{c}--- \\
\end{array}$ & 7 \\
\hline & & $\begin{array}{c}\text { Triangular } \\
\text { pointed }\end{array}$ & $\begin{array}{ll}--- \\
\end{array}$ & 8 \\
\hline & & Beaked & ---- & 9 \\
\hline \multirow[t]{3}{*}{18} & \multirow{3}{*}{$\begin{array}{c}* * \text { Node: Size of } \\
\text { bud } \\
\text { (Measured from } \\
\text { base of bud to } \\
\text { the tip) }\end{array}$} & $\begin{array}{c}\text { Small }(6 \\
\text { mm or less })\end{array}$ & 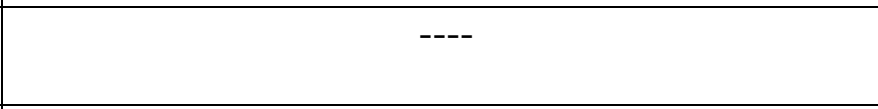 & 3 \\
\hline & & $\begin{array}{l}\text { Medium } \\
(6-9 \mathrm{~mm})\end{array}$ & $\begin{array}{l}\text { TNAU Sugarcane Si. 6, TNAU Sugarcane Si. 7, } \\
\text { Co 86032, Co 99004, Co 99006, Co 94008, Co } \\
94012 \text { and Co 2001-15. }\end{array}$ & 5 \\
\hline & & $\begin{array}{l}\text { Large }(9 \\
\text { mm or } \\
\text { more })\end{array}$ & TNAU Sugarcane Si. 8 and Co 2001-13 & 7 \\
\hline \multirow[t]{3}{*}{19} & \multirow[t]{3}{*}{$\begin{array}{l}* * \text { Node: Bud } \\
\text { groove }\end{array}$} & Absent & $\begin{array}{l}\text { TNAU Sugarcane Si. 7, Co 86032, Co 99004, Co } \\
\text { 94012, } \\
\text { Co 2001-13 and Co 2001-15. }\end{array}$ & 1 \\
\hline & & Shallow & $\begin{array}{l}\text { TNAU Sugarcane Si. 6, TNAU Sugarcane Si. 8, } \\
\text { Co } 99006 \text { and Co } 94008 .\end{array}$ & 3 \\
\hline & & Deep & ---- & 5 \\
\hline
\end{tabular}


Int.J.Curr.Microbiol.App.Sci (2017) 6(12): 509-518

\begin{tabular}{|c|c|c|c|c|}
\hline \multirow[t]{2}{*}{20} & \multirow{2}{*}{$\begin{array}{l}\text { **Node: Bud } \\
\text { cushion } \\
\text { (Space between } \\
\text { bud base and } \\
\text { leaf scar) }\end{array}$} & Absent & $\begin{array}{c}\text { TNAU Sugarcane Si. 7, Co 94008, Co } 94012 \\
\text { and Co 2001-13. }\end{array}$ & 1 \\
\hline & & Present & $\begin{array}{l}\text { TNAU Sugarcane Si. 6, TNAU Sugarcane Si. 8, } \\
\text { Co } 86032 \text {, } \\
\text { Co } 99004, \text { Co } 99006 \text { and Co 2001-15. }\end{array}$ & 9 \\
\hline \multirow[t]{3}{*}{21} & \multirow{3}{*}{$\begin{array}{l}* * * \text { Node: Bud } \\
\text { tip in relation to } \\
\text { growth ring }\end{array}$} & $\begin{array}{l}\text { Below } \\
\text { growth ring }\end{array}$ & $\begin{array}{l}\text { TNAU Sugarcane Si. } 7 \\
\end{array}$ & 1 \\
\hline & & $\begin{array}{l}\text { Touching } \\
\text { the ring }\end{array}$ & $\begin{array}{l}\text { TNAU Sugarcane Si. 6, TNAU Sugarcane Si. 8, } \\
\text { Co 86032, Co 99004, Co 94008, Co 94012, Co } \\
\text { 2001-13 and } \\
\text { Co 2001-15. }\end{array}$ & 3 \\
\hline & & $\begin{array}{l}\text { Above } \\
\text { growth ring }\end{array}$ & Co 99006 & 5 \\
\hline \multirow[t]{2}{*}{22} & \multirow{2}{*}{$\begin{array}{l}* * \text { Node: } \\
\text { Prominence of } \\
\text { growth ring }\end{array}$} & $\begin{array}{l}\text { Weak (Not } \\
\text { swollen) }\end{array}$ & Co 86032 & 1 \\
\hline & & $\begin{array}{c}\text { Strong } \\
\text { (Swollen) }\end{array}$ & $\begin{array}{l}\text { TNAU Sugarcane Si. 6, TNAU Sugarcane Si. 7, } \\
\text { TNAU Sugarcane Si. 8, Co 99004, Co 99006, Co } \\
\text { 94008, } \\
\text { Co 94012, Co 2001-13 and Co 2001-15. }\end{array}$ & 9 \\
\hline \multirow[t]{3}{*}{23} & \multirow{3}{*}{$\begin{array}{l}\text { *Node: Width } \\
\text { of root band } \\
\text { (Opposite to } \\
\text { bud) }\end{array}$} & Narrow & ---- & 3 \\
\hline & & Medium & $\begin{array}{l}\text { TNAU Sugarcane Si. 6, TNAU Sugarcane Si. 7, } \\
\text { TNAU Sugarcane Si. 8, Co 86032, Co 99004, Co } \\
\text { 99006, } \\
\text { Co 94008, Co 94012, Co } 2001-13 \text { and Co } 2001- \\
15 .\end{array}$ & 5 \\
\hline & & Broad & 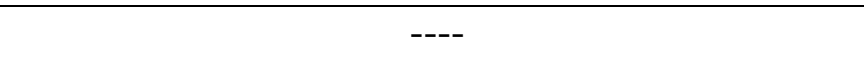 & 7 \\
\hline \multirow[t]{2}{*}{24} & \multirow[t]{2}{*}{$\begin{array}{l}* * \text { Internode: } \\
\text { Cross section }\end{array}$} & Round & $\begin{array}{l}\text { TNAU Sugarcane Si. 6, TNAU Sugarcane Si. 7, } \\
\text { TNAU Sugarcane Si. 8, Co 99004, Co 94008, Co } \\
\text { 94012, } \\
\text { Co 2001-13 and Co 2001-15. }\end{array}$ & 1 \\
\hline & & Oval & Co 86032 and Co 99006 & 2 \\
\hline \multirow[t]{2}{*}{25} & \multirow[t]{2}{*}{$\begin{array}{l}* * \text { Internode: } \\
\text { Pithiness }\end{array}$} & Absent & $\begin{array}{l}\text { TNAU Sugarcane Si. 7, Co 86032, Co 99006, Co } \\
2001-13 \text { and Co 2001-15. }\end{array}$ & 1 \\
\hline & & Present & $\begin{array}{c}\text { TNAU Sugarcane Si. 6, TNAU Sugarcane Si. 8, } \\
\text { Co 99004, Co 94008 and Co 94012. }\end{array}$ & 9 \\
\hline \multirow[t]{4}{*}{26} & \multirow{4}{*}{$\begin{array}{l}\text { **Plant: } \\
\text { Number of } \\
\text { millable canes } \\
\text { (NMC) per } \\
\text { stool }\end{array}$} & Low $(<3.0)$ & 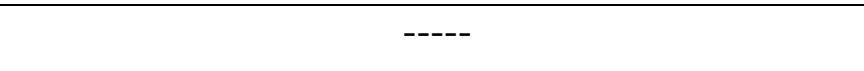 & 3 \\
\hline & & $\begin{array}{l}\text { Medium } \\
(3.0-5.0)\end{array}$ & $\begin{array}{l}\text { TNAU Sugarcane Si. 6, TNAU Sugarcane Si. } 7 \text {, } \\
\text { Co 99004, Co 99006, Co 94008, Co 2001-13 and } \\
\text { Co 2001-15. }\end{array}$ & 5 \\
\hline & & $\begin{array}{c}\text { High } \\
(5.1-7.0) \\
\end{array}$ & TNAU Sugarcane Si. 8, Co 86032 and Co 94012. & 7 \\
\hline & & $\begin{array}{l}\text { Very high } \\
(>7.0)\end{array}$ & ---- & 9 \\
\hline 27 & **Plant: Cane & Short & ---- & 3 \\
\hline
\end{tabular}




\begin{tabular}{|c|c|c|c|}
\hline height & $(<1.75 \mathrm{~m})$ & & \\
\cline { 3 - 5 } & $\begin{array}{c}\text { Medium } \\
(1.75-2.5 \\
\mathrm{m})\end{array}$ & $\begin{array}{c}\text { TNAU Sugarcane Si. 6, Co 99006, Co 94008 and } \\
\text { Co 2001-15. }\end{array}$ & 5 \\
& Tall & TNAU Sugarcane Si. 7, TNAU Sugarcane Si. 8, & 7 \\
& $(2.6-3.25$ & Co 86032, Co 99004, Co 94012 and Co 2001-13. & \\
\cline { 3 - 5 } & Very tall & ---- & 9 \\
\cline { 3 - 5 } & $(>3.25 \mathrm{~m})$ & & \\
\hline
\end{tabular}

* Monomorphic, ** Dimorphic, ***Polymorphic

Cuenya and Mariotti (1984) considered canes that deviate from erectness by more than 60 degrees as not acceptable. Elahi and Ashraf (2001) characterized six sugarcane varieties using three types (ascending transitional, dentoid and deltoid) of inner auricle. Auricle presence was used for classification of rice varieties by Singh et al., (2004) and Madhavilatha and Suneetha (2005). Sankaranarayanan et al., (1986) recognized sheath as loose, medium and tight sheath.

Swollen type of growth ring was also recorded by Akhtar et al., (2006) in GT-1, GT-7, GT-11 and F-134, and Elahiand Ashraf (2001) in CP84-1198, CP85-1491, CP881165, CP89-846, TCP86-3368 and CP77-400. Artschwager and Brandes (1958) described growth ring as a narrow zone separating the roots band from the internode above and it runs horizontally but often curves slightly upward above the bud. Chandran (2011) observed the rind wax was very prominent in eight clones among fifteen observed by him.

Almeida and Crocorno (1994a) also stated that the width of the medium leaf blade, dewlap shape, ligule and sheath auricles are the outstanding characters of value for identification of different sugarcane cultivars. Pisdtelli (1994) reported that the most important exomorphological characteristics of sugarcane varieties are shape, colour and wax of aerial organs. Almeida and Crocorno (1994b) reported that the most outstanding, organographic characters of the sugarcane stalk were the bud shape.

It can be inferred from the present study morphological characters called, descriptors for sugarcane were presented for identification of 10 cultivars and distinct morphological markers were identified for sugarcane cultivars. This could be useful for identification of cultivars in the field.

\section{Acknowledgments}

The authors are grateful to Sugarcane Research Station, Sirugamani and Sugarcane Breeding Institute, Coimbatore for providing the seed material of the germplasm and Department of Seed Science and Technology, Agricultural College and Research Institute, Madurai for providing field facilities for the conduct of the given study.

\section{References}

Akhtar, M., M. Jamil., and Ahmad, S. 2006. Agronomic traits and morphological characteristics of some exotic varieties of sugarcane. Pak. J. Agric. Res. 19(4): 70-77.

Almeida, de M., and Corcomo, O.J. 1994a. Organografia dc dez cultivares de canade-acucar (Saccharum spp), I, COI. MO. Rev. de Agriculture/. Piracicaba. 69: 41-65.

Almeida, de M., and Corcomo, O.J. 1994b. 
Organografia de dez cultivares de canade-acucar (Saccharum spp.), II, FOLHA. Rev. de Agricultura. Piracicaba. 69: 161-182.

Artschwagar, E., and Brandes, E.W. 1958. Sugarcane (Saccharum officinaram) origin classification and description of representative clones. In: Agriculture Hand book no. 122. USDA, US. Government Priority Office, Washington. Pp. 48-52.

Barber, C.A. 1919. Studies in Indian sugarcane. No. 4. Tillering or underground branching. Mem. Deptt. Agric. India. Bot. Sec. 10: 39-153.

Chandran, K. 2011. Characterization of exotic commercial hybrid clones of sugarcane. Ind. J. Plt. Genet. Res. 24(3): 271-276.

Cowgill, H.B. 1917. A method of identification and description of sugarcane cultivars and its application to types grown in Portu Rice. J. Depdt. Agric. Porto Rico. 1: No. 3.

Cuenya, M.I., and Maiott, J.A. 1984. Selection for erectness in sugarcane hybrid progenies. Sugarcane. 3: 1-15.

Elahi, N.N., and Ashraf, M. 2001. A comparative study of morphological characters of six sugarcane verities. Pak. J. Bot. 33: 503-516.

Grassl, C.O. 1956. The morphology of the grass spikelet with special reference to Saccarum. In: Proc. Int. Sugarcane Tech. 9: 764-780.

Madhavilatha, L., and Suneetha, Y. 2005. Studies on genetic divergence in rice germplasm lines. Madras Agric. $J$. 92(1-3): 12-16.

Piscitelli, F.R. 1994. Principales caracteres exonrorfologicos dc los cuttivres de cana de azucar: TUG 80- 7 Y TUC 834. Revista Industraly Agricola de / ucuman Tomo. 11: 49-57.

Sankaranarayanan, P., Natarajan, B.V., and Marimuthummal, S. 1986. Sugarcane varieties under cultivation in India. Their morphological descriptions and agricultural characteristics. ICAR, New Delhi. Pp. 3-53.

Singh, R.K., Sharma, R.K., Singh, A.K., Singh, V.P., Singh, N.K., Tiwari, S.P., and Mohapatra, T. 2004. Suitability of mapped sequence tagged microsatellite site markers for establishing distinctness, uniformity and stability in aromatic rice. Euphytica. 135: 135-143.

\section{How to cite this article:}

Chidambaram, K. and Sivasubramaniam, K. 2017. Morphological Characterization and Identification of Morphological Markers for Selected Sugarcane (Saccharum spp.) Cultivars. Int.J.Curr.Microbiol.App.Sci. 6(12): 509-518. doi: https://doi.org/10.20546/ijcmas.2017.612.062 